\title{
Interaction of arginine-based cationic surfactants with lipid membranes. An experimental and molecular simulation study
}

\author{
João A. S. Almeida ${ }^{a}$, Maria C. Morán ${ }^{a}$, Maria R. Infante ${ }^{b}$, and Alberto A. C. C. Pais ${ }^{a_{*}}$ \\ ${ }^{\mathrm{a}}$ Chemistry Department, University of Coimbra, \\ Rua Larga, 3004-535 Coimbra, Portugal \\ ${ }^{\mathrm{b}}$ Institut de Química Avançada de Catalunya, IQAC-CSIC, \\ Jordi Girona 18-26, 08034-Barcelona, España \\ E-mail: pais@qui.uc.pt
}

This work is dedicated to Prof. António M. d'A. Rocha Gonçalves, on the occasion of his $70^{\text {th }}$ birthday: to the teacher, scientist and academic

\begin{abstract}
The effect of two arginine-based cationic surfactants, arginine $N$-lauroyl amide dihydrochloride and arginine $O$-lauroyl ester dihydrochloride, upon dipalmitoylphosphatidylcholine liposomes, is addressed in this work. Some aspects concerning the synthesis of these surfactants are also presented in some detail.

Differential scanning calorimetry, as well as fluorescence anisotropy using several probes, is used to assess the relative effects on the lipid model membranes. In spite of the structural similarity among these molecules, which differ only by the group that connects the polar head to the tail, experimental results suggest differences in their behavior.

Molecular dynamics simulation provides significant insight into the molecular mechanism that governs this interaction. A rationale is provided in terms of lipid-surfactant hydrogen bonding and the consequent positioning of the surfactant molecule within the bilayer.
\end{abstract}

Keywords: Arginine-based cationic surfactants, DPPC liposomes, membrane organization

\section{Introduction}

Surfactants have been employed with success in a wide variety of fields and, due to their surface properties, have been subjected to intense study in systems involving interaction with membranes. This class of compounds combines at least one hydrophilic head group and one hydrophobic tail in the same molecule, and it is well known that the nature and structure of these groups significantly influence the behavior of such molecules. Some biological toxicity has been 
recognized in the traditional surfactants, hence limiting their applications. ${ }^{1,2}$ However, the synthesis of new biocompatible surface active molecules reopens interest in this class of compounds, and their relative actions upon biological membranes is an important issue that needs to be addressed.

In this work, two arginine-derivative surfactants, arginine- $N$-lauroyl amide dihydrochloride (ALA) and arginine-O-lauroyl ester dihydrochloride (ALE), are studied. These surfactants, previously synthesized by some of the authors, ${ }^{3,4}$ represent two biocompatible surfactants with very similar structures, differing only in the group that connects the polar head to the hydrophobic tail of the molecule. As schematically represented in Figure 1 (dotted squares), this difference corresponds to an amide and an ester functional group for the ALA and ALE surfactants, respectively.

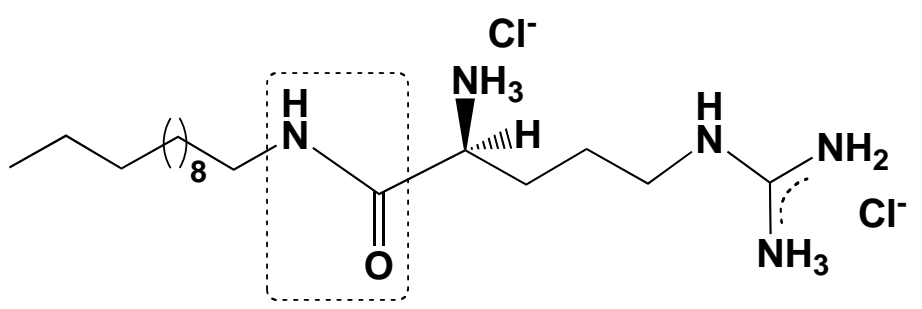

ALA

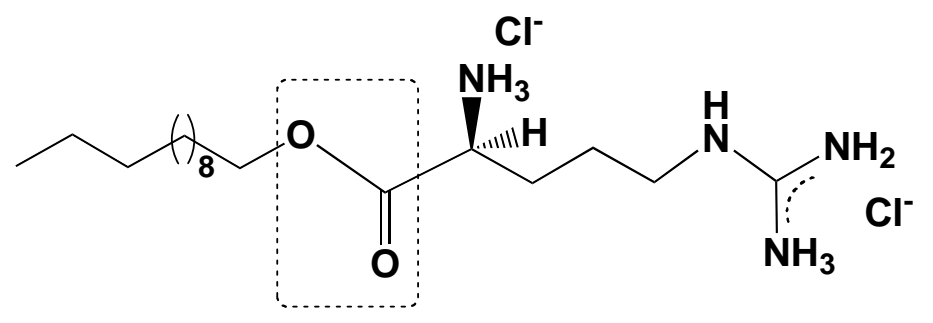

ALE

Figure 1. Chemical structure of the arginine- $N$-lauroyl amide dihydrochloride (ALA) and arginine-O-lauroyl ester dihydrochloride (ALE) derivatives.

Regarding these surfactants, a systematic study on their antimicrobial properties with different tail length was previously reported. ${ }^{5}$ This study showed that the efficiency of singlechain surfactants is affected by their alkyl chain length, with a maximum effect for the 12 carbon tail surfactants.

Over the past decade, developments on enzyme technology in non-conventional media have opened new possibilities for the synthesis of surfactants from natural sources. ${ }^{6}$ As such, the 
interest in amino acid-based surfactants lies also in their synthesis, which can be achieved by resorting to biotechnological methods. Some aspects concerning the synthesis of the relevant surfactants are addressed in the following section.

Lipid bilayers play a key role in the regulation of in vivo barriers, and surfactants are known to influence their organization ${ }^{7,8}$ and permeability. ${ }^{9}$ For the membrane interaction studies, dipalmitoylphosphatidylcholine (DPPC) liposomes were used as model lipid bilayers. This simple and well characterized model, has several advantages over the use of more complex model systems. Firstly, it allows work with a minimum amount of compound, which is relevant for assessing new synthetic chemicals. It is also suitable for the screening of a large number of conditions, since the comparitive studies rely on simple methodologies with relatively low variability. Additionally, acting as a standard, makes it easier to compare with other results in the literature.

Differential scanning calorimetry (DSC) and fluorescence anisotropy are used to study the effect of ALA and ALE surfactants upon DPPC membranes. Both techniques have been widely employed in studies of interactions relevant to liposome model systems. ${ }^{10-12}$ DSC provides a systematic tool for assessing the thermodynamic properties of the system, and allows inspection of the respective phase transitions. In turn, fluorescence anisotropy, including the use of a series of fluorescent probes, is used to establish the gradient of order across the bilayer thickness under the influence of the surfactants.

In spite of the structural similarity that exists between the two surfactants under discussion, our experimental results suggest that the effect upon DPPC liposomes differs significantly. Note also that these surfactants display different properties, such as critical micellar concentration (1.8 and $5 \mathrm{mM}$ for ALA and ALE, respectively ${ }^{5}$ ) and surface tension (37 and $30 \mathrm{mN} / \mathrm{m}$ for ALA and ALE, respectively ${ }^{5}$ ). A better understanding of the systems, in terms of the structural features of these molecules, is provided by computer simulations. This, with special emphasis on molecular dynamics (MD), has been used extensively in the last decade to describe lipid membranes in terms of their structure and dynamics. ${ }^{13-15}$ Recent work includes studies on spontaneous aggregation of phospholipids into bilayers, ${ }^{16}$ structural properties of mixed bilayers, ${ }^{17}$ pore and domain formation, ${ }^{18}$ and interaction and insertion of different molecules within lipid bilayers. ${ }^{19}$ In this work, the MD technique is shown to be very effective for the study of several aspects of the interaction between DPPC-bilayers and surfactants.

Results discussed below, both experimental and simulational, reveal that seemingly unimportant aspects of the molecular structure can modulate the interaction with lipid membranes, and influence the behavior of these membranes.

\section{Enzymatic synthesis of arginine $\boldsymbol{N}$-alkyl amide and arginine $\boldsymbol{O}$-alkyl ester derivatives}

Amide and ester bond formation between the carboxyl group of arginine and the fatty amine or alcohol is a primary concern in the synthesis of these biocompatible surfactant molecules, and proteases are the candidates of choice as biocatalyst. Primary specificity and availability are criteria which must be considered to select the most suitable. ${ }^{20,21}$ Papain accepts a wide range of 
amino acids at $\mathrm{P} 1$ but needs hydrophobic residues in the P2 position. According to the general nomenclature of cleavage site positions of the substrate, as formulated by Schechter and Berger, ${ }^{22}$ the site is denoted by P1-P1', and the numbering incremented in the $N$-terminal direction of the cleaved peptide bond as $\mathrm{P} 2, \mathrm{P} 3, \mathrm{P} 4$, etc. On the carboxyl side, the numbering is similarly incremented as P2', P3', P4', etc. In the present case, in which single amino acids are used, the requirement of hydrophobicity in the P2 position is easily fulfilled by using $N^{\alpha}$ protecting groups such as benzyloxycarbonyl (Z) or tert-butyloxycarbonyl (Boc). These proteases are of the serine and cysteine type, respectively, and amide and ester bond formation can be performed under kinetic control. Hence, $N^{\alpha}$-benzyloxycarbonyl-arginine methyl ester (ZArg-OMe) was selected as acyl-donor substrate (Figure 2). Regarding the nucleophile, papain can accept a wide range of substrate acceptors ${ }^{23,24}$ but long chain aliphatic amines and alcohols have not yet been explored.

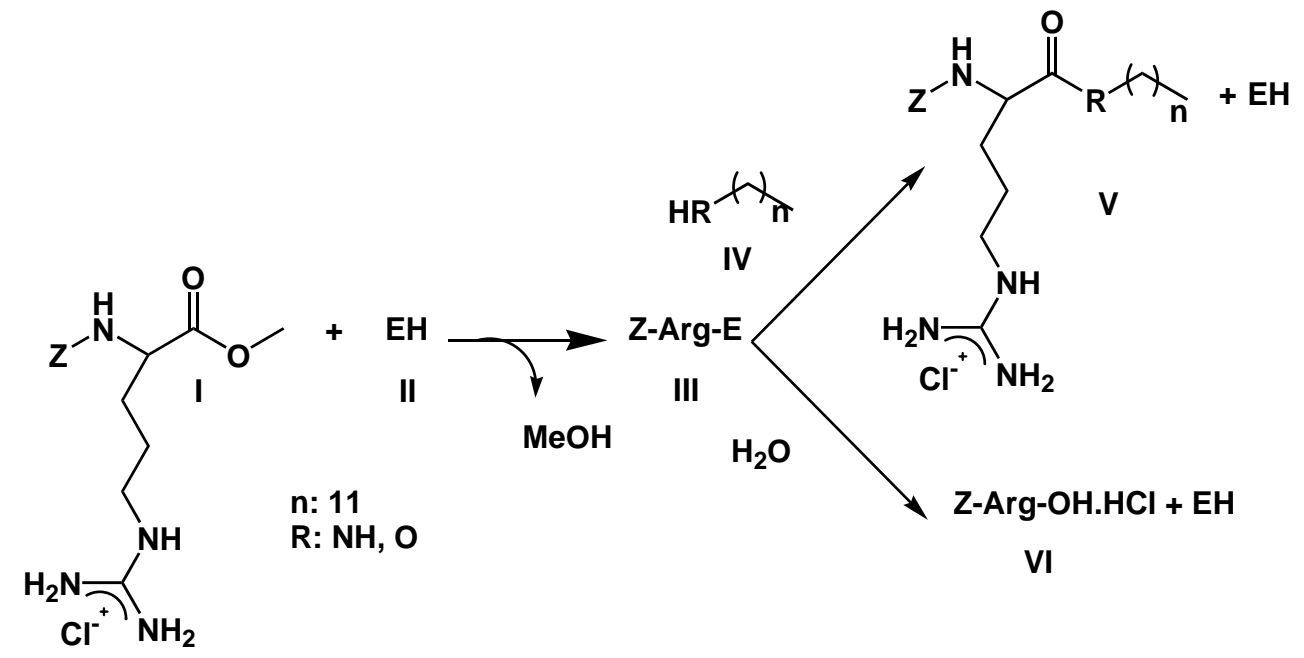

Figure 2. Reaction pathway for the synthesis of arginine alkyl amide and alkyl ester derivatives: Reaction between Z-Arg-OMe (I) and fatty amine or alcohol catalyzed by papain deposited onto a solid support: (I) acyl-donor, (II) protease, (III) acyl-enzyme, (IV) nucleophile, (V) $N^{\alpha}$ benzyloxycarbonyl-arginine $N$-alkyl amide or ester, and (VI) hydrolyzed substrate.

Papain is a cystein proteinase that has to be activated by reducing agents such as thiols, and is sensitive to oxidants. Additives, for example 2-mercaptoethanol or DTT, are commonly used as both activators and scavengers.

A low water-content system, like an organic medium, is usually selected to provide good solubility for the hydrophobic moiety (fatty amine and alcohol) and minimize the ester hydrolysis of the acyl-donor. The choice of the solvent is crucial to allow the solubilisation of both the hydrophobic and hydrophilic (acyl-donor) components. Among the solvents compatible with enzymatic activity, acetonitrile adequately matches that requirement. In the case of arginine 
alkyl esters, an ester type bond has to be formed between the carboxyl group of arginine and the hydroxyl group of a fatty alcohol.

Under optimized reaction conditions, the enzymatic synthesis of $N^{\alpha}$-benzyloxycarbonylarginine $N$-dodecyl amide (Z-Arg-NH- $\left.\mathrm{C}_{12}\right)$ and $N^{\alpha}$-benzyloxycarbonyl-arginine $O$-dodecyl ester $\left(\mathrm{Z}-\mathrm{Arg}-\mathrm{O}-\mathrm{C}_{12}\right)$ is scaled up to obtain some grams of each compound. Reaction conditions are summarized in Figure 2, while Table 1 presents some typical values of reaction yields and overall product yields. Also included in this Table are the reaction time, and deprotection and purification percentages obtained for each compound.

Table 1. Typical product and overall yields in the preparative enzymatic synthesis of arginine alkyl amide and alkyl ester derivatives (adapted from reference 3)

\begin{tabular}{ccc}
\hline & Z-Arg-NH-C & \\
\hline Reaction time (h) & 24 & Z-Arg-O-C $_{12}$ \\
Acyl-donor hydrolysis (\%) & 12 & 96 \\
Product yield (\%) & 86 & 7 \\
Overall product yield (\%), 99.9\% pure & 53 & 90 \\
\hline
\end{tabular}

The clean reaction mixtures obtained with the method described above leads to ready product purification. At the end of the synthesis, the reaction mixture consists only of product, excess of fatty amine or alcohol and hydrolysis product of the acyl-donor (Z-Arg-OH), which can be easily separated by either crystallization or chromatography.

In fact, there is significant interest in this enzymatic synthesis, in comparison to the classical approach. Previously published work indicates that the preparation of arginine $N$-alkyl amide derivatives by the classical process results in low global reaction yield (ranging from 20 to 30\%), due to the removal of byproducts formed from the coupling reagent during the purification process. $^{25}$ Also, the chemical synthesis of arginine alkyl ester derivatives requires higher temperatures and strong acid conditions, which are not always compatible with the stability of the amino acid. ${ }^{26,27}$

\section{Results and Discussion}

Many compounds have shown, in the past, the ability to directly or indirectly influence biological membrane properties and, consequently, the efficacy of drugs to pass through the membrane. Due to differences between these substances and phospholipid molecules, several changes can be induced in the membrane. Changes in conformation of alkyl tails (trans-gauche), curvature, microheterogeneity (phase separation, domain formation), thickness, surface potential and hydration of head groups, are the main mechanisms behind the interaction effect. ${ }^{28}$

Thermotropic phase transitions, the basis of calorimetric experiments, are important physicochemical properties in the analysis of drug-membrane interactions. As such, the effect of 
ALA and ALE surfactants on the thermotropic behavior of DPPC liposomes was firstly addressed using DSC.

Figure 3 displays the thermograms obtained for DPPC liposomes in the presence of various amounts of ALA, in the $0-30 \mathrm{~mol} \%$ range. Mean values of $\mathrm{T}_{\text {onset }}$ (or $\mathrm{T}_{\text {peak }}$, when more convenient), for a minimum of three repetitions, are summarized in Table 2.

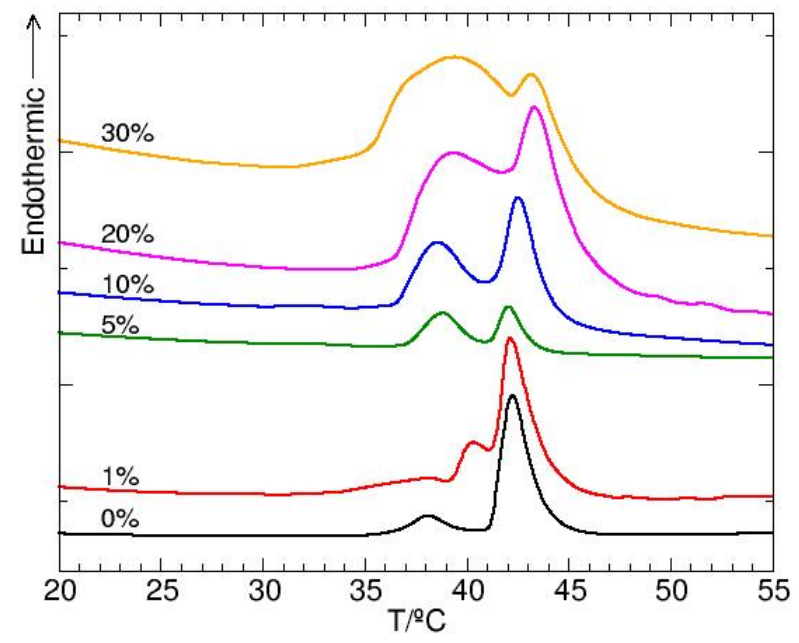

Figure 3. DSC thermograms of DPPC liposomes in the presence of various amounts of ALA (in $\mathrm{mol} \%$ ).

In the control curve (Figure 3), with no addition of surfactant, we observe the two transitions characteristic of this model. The pre-transition $\left(\mathrm{T}_{\text {onset }}=37{ }^{\circ} \mathrm{C}\right)$ corresponding to an interconversion of two solid-ordered phases $\left(\mathrm{L}_{\beta}{ }^{\prime} \rightarrow \mathrm{P}_{\beta}{ }^{\prime}\right)$, and the main transition $\left(\mathrm{T}_{\text {onset }}=42{ }^{\circ} \mathrm{C}\right)$ corresponding to a highly cooperative change to a liquid-disordered phase $\left(\mathrm{P}_{\beta}{ }^{\prime} \rightarrow \mathrm{L}_{\alpha}\right)$, reflected by a decrease in the conformational order of the phospholipid alkyl chains. ${ }^{28}$

When $1 \%$ of ALA is added, the thermogram displays an additional structure at a temperature lower than that of the main phase transition of DPPC. As the amount of ALA increases, the value of temperature corresponding to the DPPC main phase transition advances slightly to the right (see values of $\mathrm{T}_{\text {peak }}$ in Table 2, and Figure 3), suggesting some increasing degree of structuring in this domain. In contrast, the transition temperature, corresponding to the new domain, moves to increasingly lower values. The displacement of this peak is accompanied by a corresponding broadening. These results indicate that the pre-transition, characteristic of these multi-lamellar vesicles, is also affected by the presence of ALA. In the concentration range under study, the respective onset values decrease from about 37 to $31^{\circ} \mathrm{C}$, and become negligible from $15 \%$ onwards. 


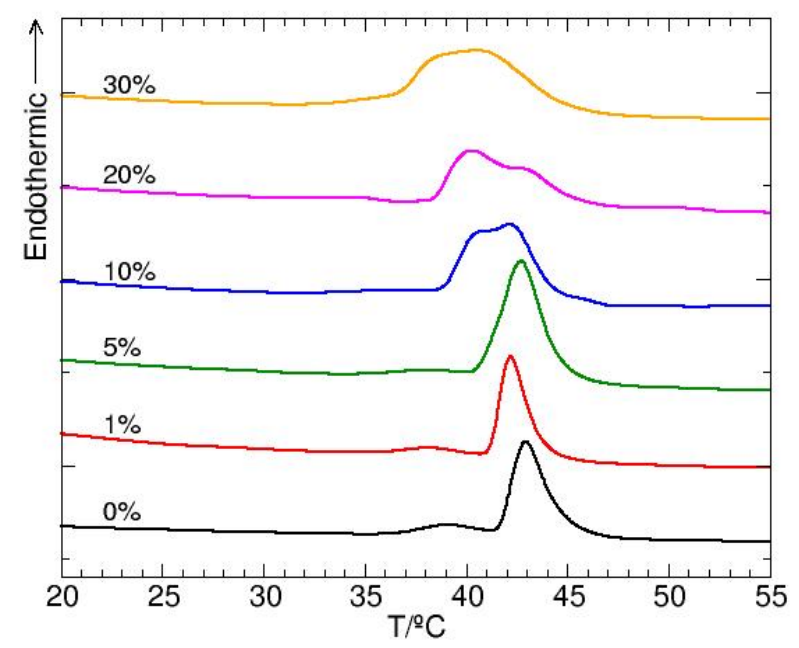

Figure 4. DSC thermograms of DPPC liposomes in the presence of various amounts (in mol\%), of the ALE surfactant.

As can be seen in Figure 4, the effect of ALE upon the phospholipid model is significantly different from that obtained with ALA. In this case, the ALE surfactant induces a broadening of the characteristic DPPC main peak.

For concentrations of ALE above $5 \mathrm{~mol} \%$, it becomes clear that more than one peak is present, although in a convoluted structure. In turn, for the maximum amount of ALE, $30 \mathrm{~mol} \%$, a unique broad structure at lower temperatures is now visible, which suggests liposome destruction. Regarding the pre-transition, it is shifted to lower temperatures as the ALE concentration increases, and vanishes for values above $20 \mathrm{~mol} \%$. Mean values of $\mathrm{T}_{\text {onset, }}$, for a minimum of three repetitions, are also gathered in Table 2.

It should be noted that the values of enthalpy corresponding to each transition cannot be directly retrieved from the figures because the amount of compound enclosed in the DSC pan is not the same for all the samples.

Table 2. $\mathrm{T}_{\text {onset }}$ and $\mathrm{T}_{\text {peak }}$ values for the phase transitions obtained in DPPC-liposomes/surfactant systems (minimum of three repetitions)

\begin{tabular}{cccccc}
\hline & \multicolumn{3}{c}{ ALA } & & \multicolumn{2}{c}{ ALE } \\
\hline & $\begin{array}{c}\text { Pre- } \\
\text { transition } \\
\mathrm{T}_{\text {onset }} /{ }^{\circ} \mathrm{C}\end{array}$ & $\begin{array}{c}\text { DPPC } \\
\text { transition } \\
\mathrm{T}_{\text {peak }} /{ }^{\circ} \mathrm{C}\end{array}$ & $\begin{array}{c}\text { left } \\
\text { transition } \\
\mathrm{T}_{\text {onset }}{ }^{\circ} \mathrm{C}\end{array}$ & $\begin{array}{c}\text { Pre- } \\
\text { transition } \\
\mathrm{T}_{\text {onset }} /{ }^{\circ} \mathrm{C}\end{array}$ & $\begin{array}{c}\text { Set of convoluted } \\
\text { transitions } \\
\mathrm{T}_{\text {onset }} /{ }^{\circ} \mathrm{C}\end{array}$ \\
\hline $0 \%$ & $36.819 \pm 0.22$ & $42.698 \pm 0.17$ & - & $36.901 \pm 0.18$ & $41.715 \pm 0.25$ \\
$1 \%$ & $35.913 \pm 0.18$ & $42.521 \pm 0.18$ & $39.252 \pm 0,14$ & $35.819 \pm 0.13$ & $41.227 \pm 0.36$ \\
$5 \%$ & $33.000 \pm 0.31$ & $42.100 \pm 0.41$ & $36.943 \pm 0.32$ & $34.448 \pm 0.26$ & $39.904 \pm 0.11$ \\
$10 \%$ & $31.406 \pm 0.34$ & $42.484 \pm 0.23$ & $35.820 \pm 0.38$ & $33.383 \pm 0.12$ & $38.941 \pm 0.14$ \\
\hline
\end{tabular}


Table 2. Continued

\begin{tabular}{|c|c|c|c|c|c|}
\hline & \multicolumn{3}{|c|}{ ALA } & \multicolumn{2}{|r|}{ ALE } \\
\hline & $\begin{array}{l}\text { Pre- } \\
\text { transition }\end{array}$ & $\begin{array}{c}\text { DPPC } \\
\text { transition }\end{array}$ & $\begin{array}{c}\text { left } \\
\text { transition }\end{array}$ & $\begin{array}{c}\text { Pre- } \\
\text { transition }\end{array}$ & $\begin{array}{l}\text { Set of convoluted } \\
\text { transitions }\end{array}$ \\
\hline & $\mathrm{T}_{\text {onset }}{ }^{\circ} \mathrm{C}$ & $\mathrm{T}_{\text {peak }} /{ }^{\circ} \mathrm{C}$ & $\mathrm{T}_{\text {onset }}{ }^{\circ} \mathrm{C}$ & $\mathrm{T}_{\text {onset }}\left({ }^{\circ} \mathrm{C}\right.$ & $\mathrm{T}_{\text {onset }} /{ }^{\circ} \mathrm{C}$ \\
\hline $20 \%$ & - & $43.425 \pm 0.12$ & $34.916 \pm 0.20$ & $30.969 \pm 0.32$ & $37.448 \pm 0.47$ \\
\hline $30 \%$ & - & $43.322 \pm 0.16$ & $34,120 \pm 0.25$ & - & $36.335 \pm 0.19$ \\
\hline
\end{tabular}

Both surfactants, ALA and ALE, cause domain formation and phase separation, which can affect membrane function in defined areas. In the case of ALA, an overall effect upon the DPPCrich domain also denotes a structuring effect of the whole membrane.

Several amino acid derivative surfactants, both single- and double-chain, have been characterized in the past, in terms of their thermotropic behavior. ${ }^{29,30}$ The interaction between arginine-based cationic surfactants with liposomes and lipid monolayers have also been studied with the aim of assessing the relationship between the antimicrobial properties of these surfactants and the perturbation of cell membranes. ${ }^{31,32}$

The DSC results from this work, discussed above, cannot be directly compared with those previous studies, and provide an important description of the phase transitions of DPPCliposomes in the presence of ALA and ALE amphiphilic molecules. However, lipid bilayers are stratified structures with a distinct trans-bilayer atom and group profile. ${ }^{33}$ In order to understand the organization and barrier function of these systems, it is important to study the molecular dynamics across the membrane. Thus, resorting to fluorescence anisotropy, and a series of fluorescent probes with ability to attach at different levels along the bilayer cross section, we further characterized the systems.

Figure 5 displays the fluorescence anisotropy results, obtained for ALA, at different depths along the DPPC chain. ALA promotes an increase of the anisotropy value at almost all depths of the bilayer, which occurs essentially in the gel $\left(\sim 30^{\circ} \mathrm{C}\right)$ phase of DPPC. At this temperature, the effect is more pronounced in the part of the chain closer to the polar heads, for this lower temperature. In the fluid phase $\left(\sim 50^{\circ} \mathrm{C}\right)$ the effect is lower, and apparently diminishes with the concentration of the surfactant. 


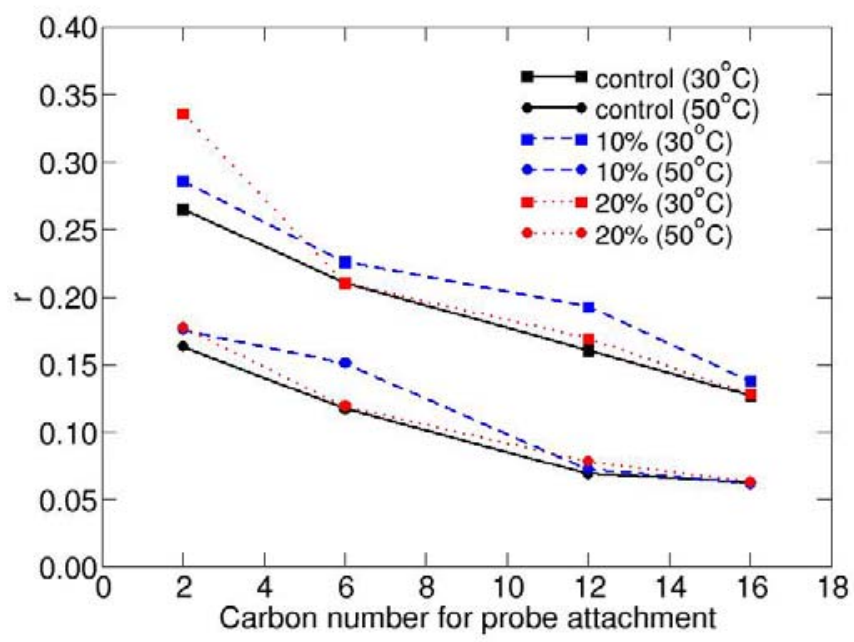

Figure 5. Fluorescence anisotropy results for DPPC liposomes in the absence and in the presence of 10 and $20 \%$ of the ALA surfactant, at the indicated temperatures.

An identical study was conducted for ALE, and the results are presented in Figure 6. This surfactant has proved to be efficient in terms of disrupting the organization of DPPC liposomes, especially at lower temperatures. The effect is more pronounced in carbon 6 , but is also visible in carbons 12 and 16.

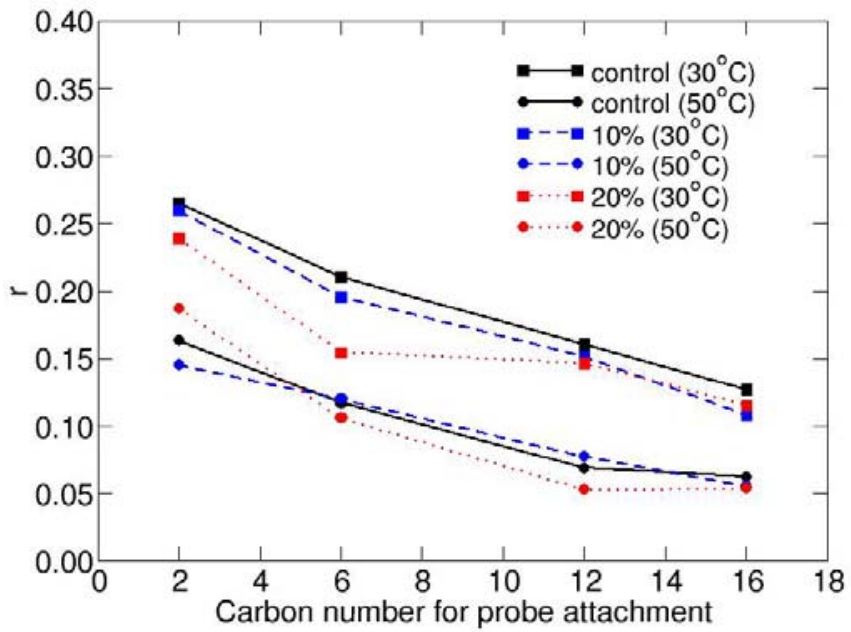

Figure 6. Fluorescence anisotropy results for DPPC liposomes in the absence and in the presence of 10 and $20 \%$ of the ALE surfactant, at the indicated temperatures.

It should be noted that the above anisotropy results are in accordance with those retrieved from DSC, pointing to corresponding effects. 
In spite of the experimental results, it is not clear which are the differences at the molecular level accounting for the dissimilar behaviors. To achieve a better understanding of the interplay of DPPC and surfactant molecules, we now refined the analysis resorting to MD results. Figures 7 and 8 display the probability for the positioning of different groups (or atoms) corresponding to the lipids, and also to the surfactants (either ALA or ALE) inserted in the membrane.

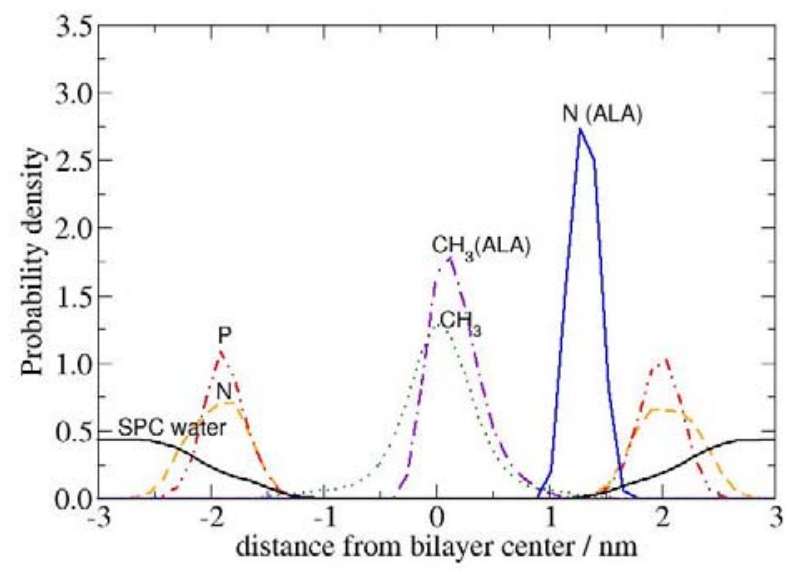

Figure 7. Probability density for the positioning of some characteristic atoms/groups for a MD run of a system comprising a DPPC bilayer in which an ALA surfactant molecule was embedded.

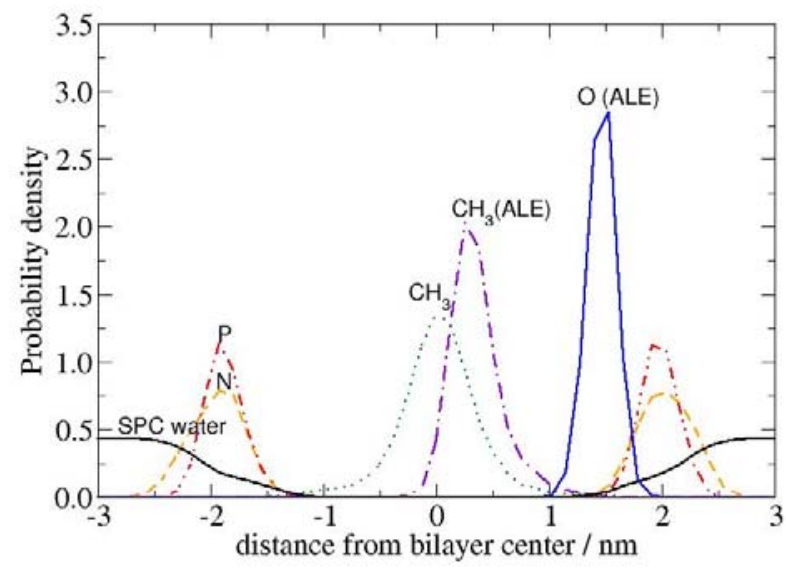

Figure 8. Probability density for the positioning of some characteristic atoms/groups for a MD run of a system comprising a DPPC bilayer in which an ALE surfactant molecule was embedded.

It is seen that the positioning of the two atoms that account for the difference between the ALA and ALE molecules is significantly lower in the former, which means that this molecule is more deeply inserted in the membrane. A direct interaction with the lipids is thus responsible for 
this differential behavior. The interaction promotes an increased organization close to the interface of the bilayer with water, in the case of ALA, as also extracted from the fluorescence anisotropy results. Specific hydrogen bonding analysis for the ALA containing system indicates a high persistence of the interaction between the $\mathrm{NH}$ group of the amide bond and the oxygen corresponding to the carbonyl groups of the fatty chains on the DPPC molecule. Obviously, a similar effect is absent in the ALE system. As a result, the methyl group at the end of the ALA tail is located, on average, in a position similar to that of the corresponding groups in the lipids. This is compatible with the fact that this surfactant determines a negligible alteration in the conformational behavior of lipids in the region close to the core of the bilayer. In contrast, the tail methyl group of ALE is above those of the lipids. In this case, a lower density region is created, and some degree of disruption arises, as also measured in fluorescence anisotropy. We note that our option was to represent, in both Figures 7 and 8, the distribution of DPPC terminal methyl groups as a single one, irrespective of the lipid belonging to the upper or lower leaflet (interdigitation makes their positioning indistinguishable). Figure 9 displays a snapshot illustrating the ALA molecule hydrogen-bonded to a DPPC lipid. Direct observation of a large series of frames extracted from the simulation clearly indicates that the motion of each of these molecules is dependent on that of the other, especially in the region of the polar head.

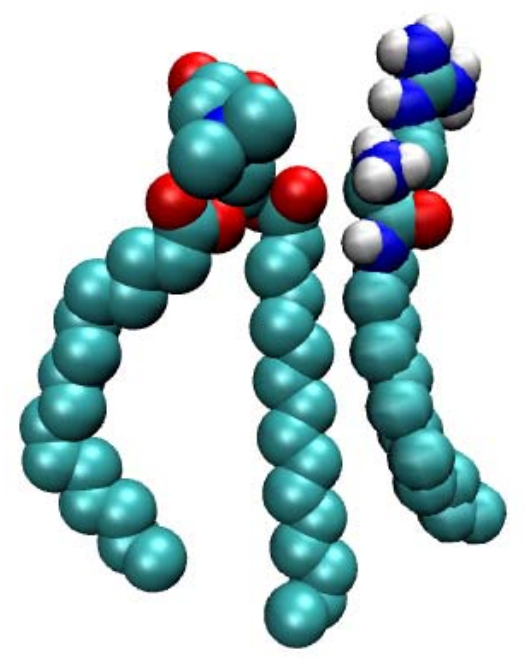

Figure 9. Snapshot containing one of the DPPC molecules of the bilayer and the ALA molecule, illustrating the H-bonding interaction.

Hydration of the alkyl chains in the bilayer is small and restricted to the polar-apolar interface. However, changes in the fluidity of the membrane, or local defects caused by the vertical positioning of specific molecules, can lead to an increase in the access of water to both the head groups and hydrocarbon chains.

Referring to the top amine groups/water radial distribution function, rdf, we assessed the degree of solvation on the polar head of each surfactant molecule. Figure 10 indicates that the 
positioning of surfactants, discussed above, significantly affects the amount of water accessible to the surfactant head, which is considerably lower in ALA, due to its retracted position.

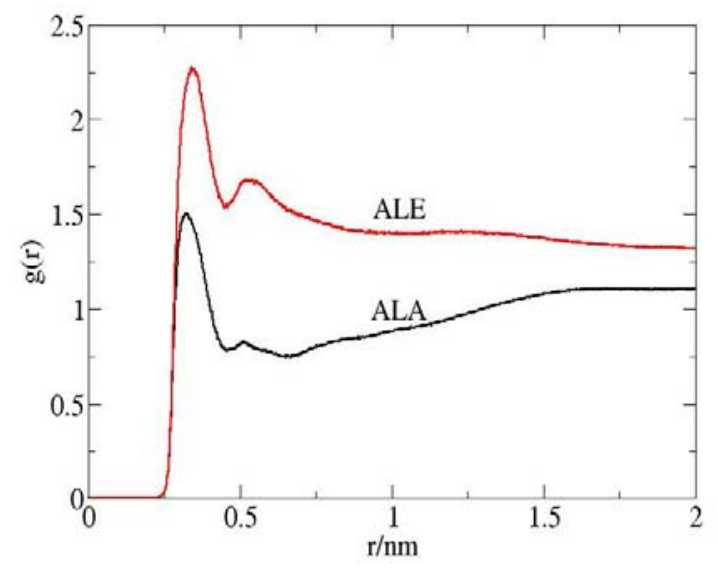

Figure 10. Top amine groups/water radial distribution function for each surfactant molecules, considered in this study, embedded in the membrane.

\section{Conclusions}

In this work we have studied the behavior of two surfactants, ALA and ALE, when inserted in DPPC lipid bilayers, and assessed the way they affect the latter. They differ only in the presence of an amide or an ester group, and are a relevant case study to check whether some minute structural changes affect the behavior and interaction of these molecules in such complex systems.

DSC results show that both the surfactants affect the thermotropic behavior of DPPC liposomes, with formation of new domains for temperatures lower than the main phase transition of DPPC. A DPPC-rich domain is, also, slightly shifted for higher temperatures in the case of ALA.

Anisotropy data further reveals the existence of relevant variations in the interaction behavior of these structurally similar molecules. ALA increases the overall degree of organization in the bilayer, while ALE induces, generally, the opposite effect.

Moreover, MD simulations reveal that the positioning of these molecules is different, with ALA more deeply inserted in the bilayer, and significantly less solvated than ALE. This stems from the formation of hydrogen bonds in the former.

Our results further suggest that variations in the structure conducted by changing the positioning of the groups responsible for this interaction within the surfactant molecule, is a way to modulate the positioning of the molecule inside the bilayer and, thus, the overall effect on the membrane, including the respective permeation properties. 


\section{Experimental Section}

General Procedures. Dipalmitoylphosphatidylcholine (DPPC), 99\% pure, was purchased from Avanti Polar Lipids and used without further purification. The probes 2-(9-anthroyloxy)stearic acid (2-AS), 6-(9-anthroyloxy)stearic acid (6-AS), 12-(9-anthroyloxy)stearic acid (12-AS) and 16-(9-anthroyloxy)palmitic acid, were obtained from Molecular Probes Inc., Eugene, OR, USA. All the fluorescence probes are 99\% pure. Solvents, dimethylformamide, methanol and chloroform were purchased from Sigma.

\section{Synthesis}

Synthesis of these surfactants followed the procedure described in reference 3. See also the specific section, above.

\section{Preparation of liposomes}

Multi-lamellar vesicles (MLVs) were prepared from DPPC according to a standard procedure, ${ }^{11}$ derived from the solvent evaporation method originally described in ref. 34. Briefly, phospholipids dissolved in a chloroform/methanol mixture were dried under reduced pressure to form a homogeneous thin lipid film deposited on the wall of a glass round-bottomed vessel. Then, slowly agitating the solution inside a bath set $10-15^{\circ} \mathrm{C}$ above the $\mathrm{T}_{\mathrm{m}}$ of DPPC, the resulting lipid was resuspended in deionized millipore water to give the desired final lipid concentration.

For the fluorescence polarization studies, instead of using water, the lipid was hydrated with a buffer solution of $10 \mathrm{mM}$ Tris-maleate, $50 \mathrm{mM} \mathrm{KCl}(\mathrm{pH} \mathrm{7})$. The resulting solution was also submitted to a program of sonication and vortexing with the aim of dispersing aggregates. This procedure does not affect the transitions of lipid bilayers, but decreases the scattered light, therefore improving fluorescence measurements. ${ }^{11,35}$

For these fluorescence studies, in order to obtain a probe/lipid molar ratio of about 1/400, a few microliters of the fluorescent probes dissolved in dimethylformamide were injected into the solution of liposomes (using an Hamilton $10 \mu$ syringe) maintained above the $T_{m}$ of DPPC under vigorous vortexing conditions. Using the same procedure, ALA and ALE surfactants from concentrated aqueous solutions were slowly incorporated in the membrane suspensions.

To assure a significant interaction between the surfactants and the liposomal structures, the final solution was left overnight inside a bath at a temperature above the $\mathrm{T}_{\mathrm{m}}$ of DPPC. Experimental tests showed that no significant differences were found from the alternative method in which phospholipids and surfactants were dissolved together, and that this was appropriate to achieve an adequate dynamical equilibrium.

The experimental protocol was specifically chosen to be compatible with the small amount of surfactants obtained via synthesis. As such, in this methodology, only a single batch of liposomes was prepared for the assessment of a set of conditions, in an experimental design that provides a highly reproducible procedure. 


\section{Differential scanning calorimetry}

DSC was performed on a Perkin Elmer Pyris 1. Volumes of $20 \mu 1$ of the liposomal suspension, containing an average of $2 \mathrm{mg}$ of DPPC, were sealed in $50 \mu \mathrm{l}$ aluminium pans. The samples were analysed by heating-cooling cycles at heating scanning rates of $10{ }^{\circ} \mathrm{C} / \mathrm{min}$ over the temperature $10-60{ }^{\circ} \mathrm{C}$, using an empty pan as reference.

The scanning rate used yielded thermograms very similar to the ones obtained with lower scanning rates, 5 and $2{ }^{\circ} \mathrm{C} / \mathrm{min}$, which means that thermodynamic equilibrium is maintained at this rate. The advantage of working with higher scanning rates is fundamentally to warrant a relatively high throughput and increase the sensitivity of the signal.

For data acquisition and subsequent analysis, the software provided by Perkin-Elmer was used. The values of $\mathrm{T}_{\text {onset }}$ for the phase transitions were determined from the intersection of the peak slopes with the baseline of the thermograms.

\section{Fluorescence anisotropy}

The rotational behavior of the $n$-(9-anthroyloxy) fatty acid fluorescence probes (2-AS, 6AS, 12AS and 16-AP) was used to obtain information about the lipid dynamic at various depths of the liposome membranes in both gel and liquid crystalline phases of DPPC. The photophysical features of these probes and their application to the study of vesicles and membranes were intensively studied in the past. ${ }^{36-39}$

All the fluorescence anisotropy measurements were made using a Perkin Elmer LS 55B fluorescence spectrophotometer, fitted with temperature control and magnetic stirrer. Temperature was measured with an accurance of $0.1{ }^{\circ} \mathrm{C}$, and the stirrer switched off during the experiments. The samples were excited at $365 \mathrm{~nm}$ and emission detected at $450 \mathrm{~nm} .^{11}$

Values of fluorescence anisotropy measurements, $r$, were obtained according to

$$
r=\frac{I_{\|}-I_{\perp} \times G}{I_{3}-2 I_{\perp} \times G}
$$

in which $I_{\|}$and $I_{\Perp}$ are the intensities of the light emitted with its polarization plane parallel and perpendicular to that of exciting beam, respectively. ${ }^{38} G$ is the grating correction factor for the optical system and is defined by the ratio between the vertically polarized emission component and the horizontal one, when the sample was excited with polarized light in the horizontal direction. $^{11}$

\section{Molecular dynamics}

Molecular Dynamics simulations were carried out under periodic boundary conditions, resorting to the GROMACS package, version 3.3.3, ${ }^{40-42}$ and the GROMOS96 $45 \mathrm{a} 3$ force field ${ }^{43}$. This force field, developed specifically for simulations of lipids and detergents, was based on GROMOS 96 $43 \mathrm{a} 1$ and extended with parameters provided in ref. 43. 
A fully hydrated DPPC bilayer was generated and equilibrated before the insertion of the surfactant molecules. A relatively small DPPC bilayer consisting of 72 DPPC molecules, equally distributed by two leaflets, was built by placing molecules in a regular grid with random rotation along the major axis. It should be noted that the size of the bilayer was chosen to be large enough to accommodate one embedded surfactant molecule, and allow the study of its interaction with the nearby lipids.

To obtain a starting configuration close to a local minimum, the DPPC bilayer hydrated with 1672 SPC water molecules was firstly subjected to an energy minimization step. After that, using the LINCS algorithm to maintain the DPPC molecular group under positional restraint conditions, the system was equilibrated for $10 \mathrm{~ns}$. The system was then left to evolve without restraints, with a time step of $2 \mathrm{fs}$. Temperature and pressure were coupled to the Berendsen external baths maintained at $323 \mathrm{~K}$ and 1 bar (in isotropic conditions), with coupling constants of 0.1 and $0.5 \mathrm{ps}$, respectively. Non-bonded interactions were computed on the basis of a neighbor list, updated every 10 steps, while Lennard-Jones and electrostatic interactions, with a cut-off distance of $1.0 \mathrm{~nm}$ and $1.8 \mathrm{~nm}$ respectively, were evaluated at each time step. The system was considered equilibrated when reaching an area per lipid, order parameters and density profiles close to the experimental results for such membranes.

In order to circumvent the timescale limitations associated with a full description of the process of insertion, the surfactants were directly incorporated in the pre-equilibrated and fully hydrated DPPC bilayer. We note that electroneutrality of the system is achieved by the inclusion of only two counterions, $\mathrm{Cl}^{-}$, pertaining to each surfactant molecule, since DPPC is a zwiterionic phospholipid. We decided to initiate the equilibration phase with the incorporation of the surfactants in the core of the bilayer, without specifically creating a cavity for its insertion. DPPC + surfactant systems were simulated under the same parameters imposed to the DPPC preequilibrated system. A minimization step was followed by an equilibration of $2 \mathrm{~ns}$, keeping DPPC and surfactant molecular groups under positional restraint conditions, and $10 \mathrm{~ns}$ without restraints. A production run of $10 \mathrm{~ns}$ was subsequently calculated, and subjected to standard analysis: atom-atom (group-group) distance probability distributions, and atom (group) density probability distributions.

\section{Acknowledgements}

J. A. S. A. gratefully acknowledges PhD grant SFRH/BD/31064/2006, Fundação para a Ciência

e Tecnologia. The authors kindly acknowledge Prof. A. S. Jurado for the use of DSC and fluorescence anisotropy equipments.

\section{References}

1. Lee, J. K.; Kim, D. B.; Kim, J. I.; Kim, P. Y. Toxicol. in Vitro. 2000, 24, 345. 
2. Jadhav, V.; Maiti, S.; Dasgupta, A.; Das, P. K .; Dias, R. S.; Miguel, M. G.; Lindman, B. Biomacromolecules 2008, 9, 1852.

3. Morán, M. C.; Clapés, P.; Infante, M. R. Biotec Biotechnol. Bioeng. 1999, 63, 333.

4. Clapés, P.; Morán, M. C.; Infante, M. R. ES Patent 9700 520, 1997.

5. Morán, C.; Clapés, P.; Comelles, F.; García, T.; Pérez, L.; Vinardell, P.; Mitjans, M.; Infante, M. R. Langmuir 2001, 17, 5071.

6. Sarney, D. B.; Vulfson, E. N. Trends in Biotechnology 1995, 13, 164.

7. Koynova, R.; Techov, B. Curr. Opin. Coll. Interf. Sci. 2001, 6, 277.

8. Xia, W. J.; Onyuksel, H. Pharm. Res. 2000, 17, 612.

9. William, A. C.; Barry, B. W. Adv. Drug Deliv. Rev. 2004, 56, 603.

10. El Maghraby, G. M. M.; Williams, A. C.; Barry, B. W. Int. J. Pharm. 2004, 276, 143.

11. Videira, R. A.; Antunes-Madeira, M. C.; Madeira, V. M. C. Chem. Phys. Lipids 1999, 97, 139.

12. de Castro, B.; Gameiro, P.; Lima, J. L. F. C.; Matos, C. Colloids Surf. A. 2001, 190, 205.

13. Feller, S. E. Curr. Opin. Colloid Interface Sci. 2000, 5, 217.

14. Forrest, L. R. ; Sansom, M. S. P. Curr. Opin. Struct. Biol. 2000, 10, 174.

15. Sabra, M. C.; Mouritsen, O. G. Methods Enzymol. 2000, 321, 263.

16. Marrink, S. J. ; Lindahl, E.; Edholm, O.; Mark, A. E. J. Am. Chem. Soc. 2001, 123, 8638.

17. Leekumjorn, S.; Sum, A. K. Biophys. J. 2006, 90, 3951.

18. Leotiadou, H.; Mark, E.; Marrink, J. Biophys. J. 2004, 86, 2156.

19. Babakhani, A.; Gorfe, A.; Kim, J. E.; McCammon, J. A. J. Phys. Chem. B. 2008, 112, 10528.

20. Bond, J.S. In Proteolytic enzymes: A practical approach. $1^{\text {st }}$ Edn; Beynon, R. J.; Bond, J. S. Eds.; IRL Press: Oxford, 1989; pp 232-240.

21. Kasche, V. In Proteolytic enzymes: A practical approach. $1^{\text {st }}$ Edn. Beynon, R. J.; Bond, J. S., Eds. IRL Press: Oxford, 1989, pp 125-143.

22. Schechter, I.; Berger, A. Biochem. Biophys. Res. Commun. 1967, 27, 157.

23. Fischer, U.; Schellenberger, V.; Mitin, Y. M.; Jakubke, H-D. In Peptide chemistry. Shiba, T.; Sakakibara, S. Eds. Protein Research Foundation: Osaka, 1987, pp 413-416.

24. Salchert, K.; Ullmann, D.; Jakubke, H-D. Pharmazie. 1996, 51, 710.

25. Piera, E.; Comelles, F.; Erra, P.; Infante, M. R. J. Chem. Soc. Perkin Trans. 2 1998, 335.

26. Braun, P.; Waldmann, H.; Vogt, W.; Kunz, H. Synlett 1991, 2, 105.

27. Selve, C.; Hamdoume, F.; Mansuy, L.; Allouch, M. J. J. Chem. Res. (S) 1992, 22.

28. Seydel, J. K. Drug-Membrane Interactions: Analysis, drug distribution, modeling. $1^{\text {st }}$ Edn. Wiley: Weinheim, 2002, pp 1-50.

29. Morán, M. C.; Pinazo, A.; Clapés, P.; Pérez, L.; Infante, M. R.; Pons, R. J. Phys. Chem. B. 2004, 108, 11080.

30. Morán, M. C.; Pinazo, A.; Clapés, P.; Infante, M. R.; Pons, R. J. Phys. Chem. B. 2005, 109, 22899. 
31. Lozano, N.; Pérez, L.; Pons, R.; Luque-Ortega, J. R.; Fernández-Reyes, M.; Rivas, L.; Pinazo, A. Colloids Surf. A 2008, 319, 196.

32. Castilho, J. A.; Pinazo, A.; Carilla, J.; Infante, M. R.; Alcina, M. A.; Haro, I.; Clapés, P. Langmuir 2004, 20, 3379

33. Mouritsen, O. G. Life - As a Matter of Fat. $1^{\text {st }}$ Edn. Springer: Berlin, 2005, pp 81-89.

34. Bangham, A. D.; Hill, M. W.; Miller, N. G. A. in Methods in Membrane Biology; Plenum, N.Y.; Vol. 1, pp 1-68.

35. Rosa, S. M. L. J.; Antunes-Madeira, M. C.; Matos, M. J.; Jurado, A. S.; Madeira, V. M. C. Biochim. Biophys. Acta 2000, 1487, 286.

36. Thulborn, K. R.; Sawyer. W. H. Biochim. Biophys. Acta 1978, 511, 125.

37. Thulborn, K. R.; Tilley, L. M.; Sawyer. W. H.; Treloar, F. E. Biochim. Biophys. Acta 1979, $558,166$.

38. Shinitzky, M.; Barenholz, Y. Biochim. Biophys. Acta 1979, 515, 367.

39. Haigh, A.; Thulborn, K. R.; Sawyer. W. H. J. Am. Chem. Soc. 1979, 18, 3525.

40. van der Spoel; Lindahl, E.; Hess, B.; Groenhof, G.; Mark, A. E.; Berendse N, H. J. C. J. Comp. Chem. 2005, 26, 1701.

41. Lindhal, E.; Hess, B.; van der Spoel. J. Mol. Mod. 2001, 7, 306.

42. Berendsen, H. J. C.; van der Spoel; van Drunen, R. Comp. Phys. Comm. 1995, 91, 43.

43. Schuler, L. D.; Daura, X.; Van Gusteren, W. F. J. Comp. Chem. 2001, 22, 1205. 